\section{Liquid handling}

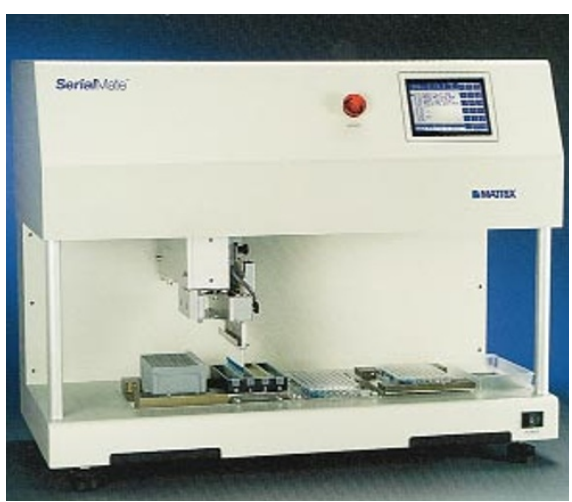

Microplate pipetting system

Available from Matrix Technologies (Hudson, NH), SerialMate is a multichannel automated pipetting workstation capable of performing various routine liquid manipulations such as reagent addition, serial dilution, microplate replication, and sample dilution. SerialMate is compatible with both shallow and deep 96-well microplates, and filtered and nonfiltered disposable tips allow pipetting in volumes from 5 to $200 \mu$ l. http://www.matrixtechcorp.com RIN: 1198

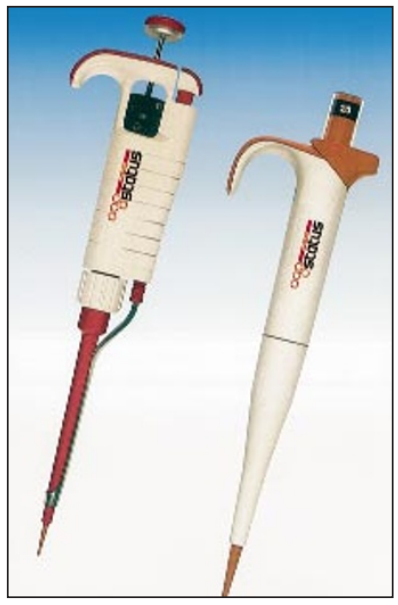

\section{Range of pipettors}

Status pipettors from Philip Harris Scientific (Lichfield, UK) are available in both singlechannel and multichannel models, with features including a tip ejector that is positioned to avoid unintentional tip ejection and that can be removed completely when working in deep, narrow tubes. There are seven variable volume pipettors with volume ranges from 0.2 to $5000 \mu \mathrm{l}$, and multichannel pipettors are available in 8 - or 12channel formats.

http://www.philipharris.co.uk/scientific/ RIN: 1199

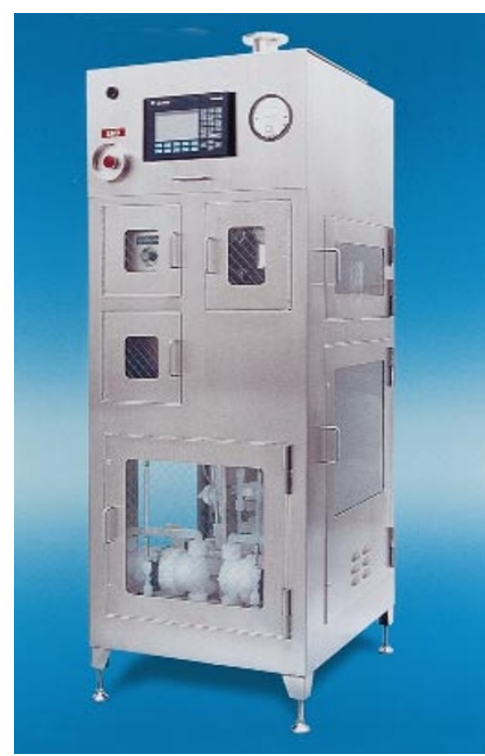

\section{Chemical dispenser}

The MEGApure 100 SS chemical dispense system by Kinetics (Santa Clara, CA), a division of US Filter, is designed to create superior particle performance and increase safety as well as being cost effective. It is ideal for dispensing acids, bases, oxidizers, and solvents, and features local and remote EMO capability and nonobtrusive drum empty detection. Options include auto changeover for the supply source and a redundant pump. http://www.kineticsgroup.com RIN: 1200

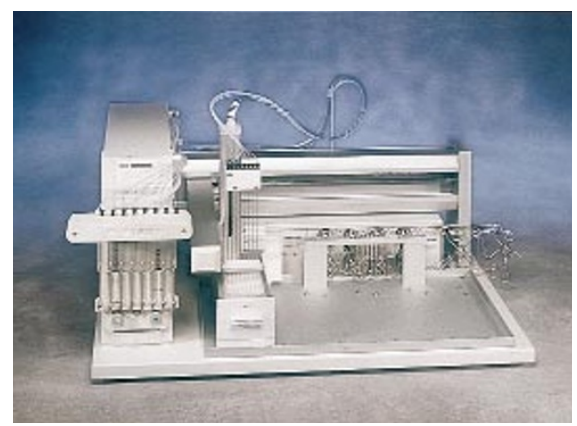

\section{Injector gadget}

Gilson's (Middleton, WI) Multiple Probe 215 with the 889 Injector allows for multipleprobe liquid handling and multiple injections onto LC and LC/MS systems. Based on Gilson's popular 215 Liquid Handler, the Multiple Probe 215 has a sevenfold higher throughput capability, and is ideal for highthroughput drug discovery and drug metabolism laboratories.

http://www.gilson.com

RIN: 1201

\section{Vectors \& expression systems}

\section{Tetracycline-regulated expression}

Invitrogen's (Carlsbad, CA) T-REx (tetracycline-regulated expression) System is the first inducible mammalian expression system to use the full-length CMV promoter, which provides the highest yields of recombinant protein in a wide range of cells. Regulating expression from this powerful promoter permits study of proteins that may have pleiotropic effects. The system uses a repressor to regulate transcription, eliminating the nonspecific effects of transactivators used by other tetracycline-regulated systems.

\section{http://www.invitrogen.com}

RIN: 1202

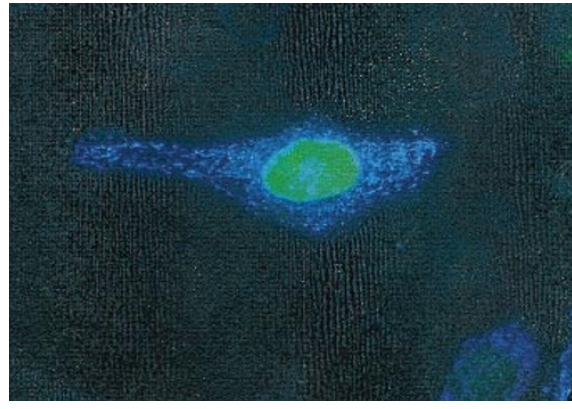

\section{Vivid vectors}

Clontech Laboratories (Palo Alto, CA) offers Living Colors Subcellular Localization Vectors for dynamic localization studies in mammalian cells by fluorescence microscopy. The vectors contain Clontech's enhanced GFP variants that can be targeted to the nucleus, mitochondria, or cytoskeleton, making the vectors convenient for studying cytoskeletal and organelle structure and function in living cells, in real time and without chemical staining. http://www.clontech.com RIN: 1203

\section{High-density cells}

Protein Sciences' (Meriden, CT) proprietary expresSF+ serum-free high-density insect cells for use in the baculovirus expression vector system are suitable for research and cGMP production of recombinant proteins. They meet FDA standards for identity and stability, and yield $10^{7}$ or more cells $/ \mathrm{ml}$ with nearly $100 \%$ viability in standard suspension cultures. And unlike other serum-free insect cells, expresSF+ cells also generate high titers of baculovirus, so a single cell line can be used for the production of recombinant baculoviruses and proteins.

http://www.proteinsciences.com RIN: 1204 